\title{
Comprometimento e promoção do sono em unidades de terapia intensiva: revisão integrativa
}

\section{Sleep impairment and sleep promotion in intensive care units: an integrative review Comprometimiento y promoción del sueño en unidades de cuidados intensivos: revisión integradora}

Florinda Laura Ferreira Rodrigues Galinha de Sá1 io https://orcid.org/0000-0002-4523-1721

Filipa Bernardes Neves Miranda² id hitps:/orcid.org/0000-0002-7945-8297

Inês Mariana da Silva Reboredo Morais ${ }^{3}$ io https://orcid.org/0000-0002-0267-7117

Margarida Albuquerque Duarte Moniz de Almeida ${ }^{4}$ io https://orcid.org/0000-0003-3668-8444

Marta Carvalho Afons0 ${ }^{5}$ io hitps://orcid.org/0000-0003-3011-4941

\section{Como citar:}

Sá FL, Miranda FB, Morais IM, Almeida MA, Afonso MC. Comprometimento e promoção do sono em unidades de terapia intensiva: revisão integrativa. Acta Paul Enferm. 2021;34:AAPE00023.

DOI

http://dx.doi.org/10.37689/actaape/2021AR00023

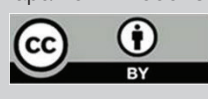

Descritores

Sono; Conforto do paciente; Unidades de terapia intensiva; Cuidados de enfermagem; Transtornos

do sono-vigília

\section{Keywords}

Sleep; Patient confort; Intensive care units; Nursing care; Sleep wake disorders

\section{Descriptores}

Sueño; Comodidad del paciente; Unidades de cuidados intensivos; Cuidados de enfermeira; Trastornos del sueño-vigilia

\section{Submetido \\ 3 de Janeiro de 2020 \\ Aceito \\ 20 de Agosto de 2020}

Autor correspondente

Florinda Laura Ferreira Rodrigues Galinha de Sá

Email: fgalinha@esel.pt

\section{Resumo}

Objetivo: Identificar, nas bases de dados científicas, as evidências que comprometem o sono e as intervenções de enfermagem promotoras do sono em Unidades de Terapia Intensiva.

Métodos: Revisão integrativa da literatura, realizada nas bases de dados MEDLINE, CINAHL e Cochrane Database of Systematic Reviews, para responder às questões norteadoras do estudo, de acordo com o formato PICo, nos meses de maio e junho de 2019. Os idiomas considerados foram o inglês, português e espanhol.

Resultados: Identificou-se 110 estudos e 12 foram considerados elegíveis para a revisão. A análise reporta que o comprometimento do sono é multicausal, sendo este influenciado sobretudo por desconforto ambiental e físico do paciente. As intervenções de enfermagem, tais como a gestão do ambiente, no âmbito do ruído e da luminosidade, o agrupamento da prestação de cuidados, o controle da dor e da ansiedade do paciente internado, são fundamentais para a qualidade do sono.

Conclusão: A produção científica evidencia que as intervenções de Enfermagem identificadas, sobretudo no âmbito da promoção do conforto ambiental e físico do paciente, contribuem para a qualidade do sono em Unidades de Terapia Intensiva, contudo a promoção do conforto psicoespiritual e social do paciente ainda é superficial, tendo em conta o foco e as prioridades nesta tipologia de internamento.

\section{Abstract}

Objective: To identify, in the scientific databases, evidence of factors that impair sleep and nursing interventions to promote sleep in Intensive Care Units.

Methods: An integrative literature review was conducted across the databases MEDLINE and CINAHL and the Cochrane Database of Systematic Reviews between May and June 2019 using the PICO framework, seeking to answer the guiding questions of the study. The languages included in the literature review were English, Portuguese, and Spanish.

Results: One hundred and ten studies were identified, twelve of which were considered eligible for review. The analysis of the articles found that sleep impairment is multicausal, being influenced mainly by the patient's environmental and physical discomfort. Nursing interventions, such as environmental management of noise and light, grouping of care procedures, and control of pain and anxiety in the hospitalized patient, are fundamental for sleep quality.

Conclusion: The academic literature shows that the nursing interventions identified, particularly those that promote the patient's environmental and physical comfort, contribute to the quality of sleep in 
Intensive Care Units. However, promotion of the patient's psychospiritual and social comfort is still lacking, given the focus and priorities of this type of hospitalization.

\section{Resumen}

Objetivo: Identificar, en bases de datos científicas, las evidencias que comprometen el sueño y las intervenciones de enfermería para promover el sueño en unidades de cuidados intensivos.

Métodos: Revisión integradora de literatura, realizada en las bases de datos MEDLINE, CINAHL y Cochrane Database of Systematic Reviews para responder las preguntas orientadoras del estudio, de acuerdo con el formato PICO, en los meses de mayo y junio de 2019. Los idiomas considerados fueron inglés, portugués y español.

Resultados: Se identificaron 110 estudios y 12 fueron considerados elegibles para la revisión. El análisis reporta que el comprometimiento del sueño es multicausal y lo que más influye en el sueño es la incomodidad ambiental y física del paciente. Las intervenciones de enfermería, tales como el manejo del ambiente respecto al ruido y a la luminosidad, el agrupamiento de la prestación de cuidados y el control del dolor y de la ansiedad del paciente internado, son fundamentales para la calidad del sueño.

Conclusión: La producción científica evidencia que las intervenciones de enfermería identificadas, sobre todo respecto a la promoción del bienestar ambiental y físico del paciente, contribuyen con la calidad del sueño en unidades de cuidados intensivos. No obstante, la promoción del bienestar piscoespiritual y social del paciente aún es superficial, teniendo en cuenta dónde se centra la atención y cuáles son las prioridades en este tipo de internación.

\section{Introdução}

O paciente internado numa Unidade de Terapia Intensiva (UTI) é frequentemente submetido a inúmeros estímulos sensoriais externos que, consequentemente, comprometem o seu sono e repouso, agravando ainda mais a sua situação de vulnerabilidade acrescida e atrasando a sua recuperaçáo. ${ }^{(1)} \mathrm{O}$ sono decorre da "redução recorrente da atividade corporal, marcada por uma diminuição da consciência (...), metabolismo diminuído (...) e sensibilidade diminuída, mas prontamente reversível a estímulos externos.”. (2) O sono divide-se em dois ciclos: REM, que corresponde ao movimento rápido dos olhos $(20-25 \%$ do tempo total do sono) e NREM, que corresponde ao ciclo de movimento náo rápido dos olhos (70-75\% do tempo total do sono). Por sua vez, o ciclo NREM subdivide-se em 4 estadios (N1, N2, N3 e N4), que correspondem a um sono cada vez mais profundo. Estes ciclos mencionados alternam entre si durante a noite, sendo que, durante o sono normal do adulto completam-se 4 a 5 destes ciclos. ${ }^{(3)}$

O paciente internado numa UTI tem a vida ameaçada por falência ou eminência de falência de uma ou mais funçóes vitais, estando dependente de meios avançados de vigilância, monitorização e terapêutica. ${ }^{(3)} \mathrm{O}$ paciente com comprometimento da qualidade do sono apresenta um sono fragmentado com várias interrupçôes e com diminuição ou ausência da fase restauradora do sono e com alteração do ritmo circadiano. ${ }^{(4)}$ Estas alteraçóes acarretam várias repercussões fisiológicas, principalmente ao nível do sistema neurológico (diminuição da memória, da atenção e tempo de resposta), circulatório (hipertensão, bradicardia e mudanças extensas nos mecanismos homeostáticos), endócrino (aumento da produção de tiroxina, noraepinefrina e cortisol; diminuição da somatotrofina; e aumento da resistência à insulina) e respiratório (diminuição da função muscular com repercussóes no desmame ventilatório). ${ }^{(4)}$ A privação do sono é ainda um fator de risco para o delirium, o que pode conduzir a uma maior morbilidade e mortalidade, aos seis meses e um ano, e consequentemente a um aumento do período de internamento e dos custos em saúde. ${ }^{(5)}$

A Enfermagem no âmbito da promoção do sono, e de acordo com os padrôes de qualidade da profissão, irá atuar no sentido da promoção da saúde, do bem-estar do paciente e na prevenção de complicaçóes, pois assume uma dimensão da humanização dos cuidados, na medida em que o Enfermeiro tem o dever de "contribuir para criar o ambiente propício ao desenvolvimento das potencialidades da pessoa". (6) Segundo a Teoria de Conforto de Kolcaba, os cuidados de enfermagem visam o conforto do paciente, sendo o conforto "a experiência imediata e holística de ser fortalecido através da satisfação das necessidades nos três tipos de conforto (alívio, transcendência e tranquilidade)", ${ }^{(7)}$ sendo que este pode ser atingido em quatro contextos da experiência: o físico (sensaçóes do corpo); o psicoespiritual (consciência interna de si mesmo); o social (relações interpessoais) e o ambiental (influências externas do contexto). ${ }^{(8)}$ 
Sendo o sono essencial para o bem-estar do ser humano, este continua a ser negligenciado nas UTI, que direcionam a sua ação principalmente para a manutenção das funções vitais do paciente. ${ }^{(9)}$ Consideramos assim, crucial, a abordagem da enfermagem na promoção do sono a partir do foco no conforto do paciente internado em UTI. Assim, constituem-se como objetivos deste artigo identificar as evidências que comprometem o sono e as intervençóes de enfermagem promotoras do sono em UTI.

\section{Métodos}

Trata-se de uma revisão integrativa da literatura (RIL) o que permite "a síntese de múltiplos estudos publicados e possibilita conclusóes gerais a respeito de uma particular área de estudo.”. (10) As questóes de investigação que nortearam a pesquisa foram: "Quais as causas do comprometimento do sono no paciente em UTI?" e "Quais as intervençóes de enfermagem para promover o sono no paciente em UTI?”. De modo a auxiliar a nossa pesquisa, tivemos por base o referencial PICo (população, fenômeno de interesse e contexto), que se encontra representado na Tabela 1 sendo que, identificamos como população $(\mathrm{P})$ o paciente crítico, como fenômeno de interesse (I) o sono e as intervençóes de enfermagem e como contexto (Co) a Unidade de Terapia Intensiva (Quadro 1).

Quadro 1. Modelo PICo para a pesquisa em base de dados de acordo com os descritores

\begin{tabular}{|c|c|c|}
\hline$P$ & 1 & Co \\
\hline $\begin{array}{l}\text { Critical Patient OR } \\
\text { Critically III }\end{array}$ & $\begin{array}{l}\text { Sleep OR Sleep Disruption } \\
\text { OR Sleep Deprivation Or } \\
\text { Sleep Disturbance AND } \\
\text { Nurs* }^{\star}\end{array}$ & $\begin{array}{l}\text { Intensive Care Unit OR } \\
\text { Intensive Care Units OR } \\
\text { Critical Care }\end{array}$ \\
\hline
\end{tabular}

A revisão foi elaborada de acordo com a estrutura: seleção do tópico de estudo; pesquisa da literatura; leitura e análise dos artigos; e organização dos resultados para descrição e discussão. ${ }^{(11)} \mathrm{A}$ pesquisa decorreu no período entre maio e junho de 2019 e teve como ponto de partida o motor de busca EBSCO, através do qual recorremos a três bases de dados CINHAL, MEDLINE e Cochrane Database of Systematic Reviews simultaneamente, de modo a eliminar duplicados. Os descritores referidos na tabela 1 foram organizados de acordo com os seguintes operadores booleanos: [(critical patient OR critically ill) AND (sleep OR sleep disruption OR sleep deprivation OR sleep disturbance) AND (nurs* OR nursing care) AND (intensive care units OR intensive care unit OR critical care)] e selecionados de acordo com os descritores específicos para cada base de dados, contribuindo assim para a sensibilidade da pesquisa.

Como critério de inclusão foram considerados: artigos primários e secundários que abordassem a temática em estudo, disponível na integra, em português, inglês e espanhol, publicados nos últimos dez anos, considerando o critério de exequibilidade analítica face ao extenso número de publicaçóes. Foram excluídos artigos que abordavam o paciente pediátrico ou neonatal em UTI pelas especificidades associadas ao padrão de sono deste tipo de população. ${ }^{(3)}$ Os artigos foram revisados de forma independente pelos autores na fase de busca e seleção, tendo-se obtido, após a leitura integral, uma amostra final de 12 artigos (Figura 1).

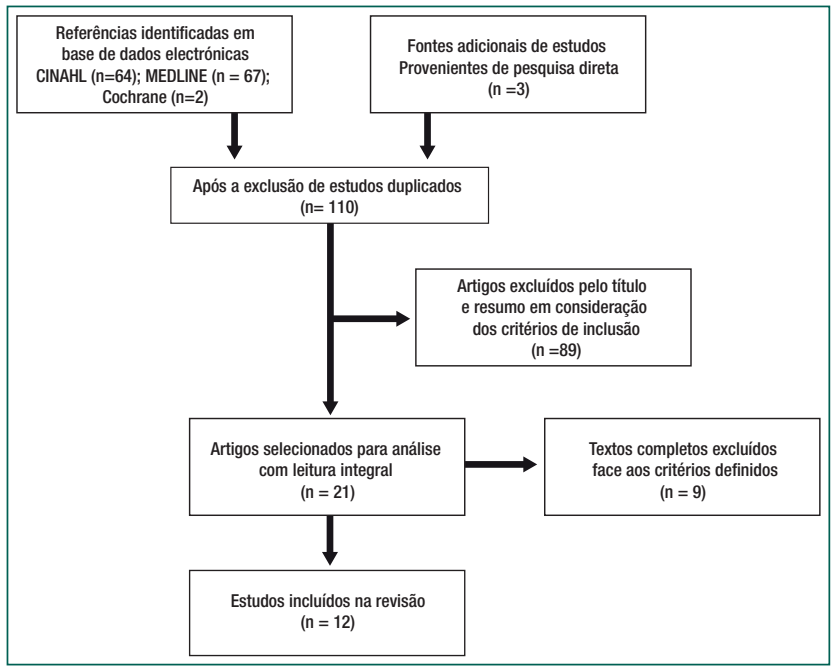

Fonte: Moher D, Liberati A, Tetzlaff J, Altman DG. Preferred reporting items for systematic reviews and meta-analyses: The PRISMA statement. Int J Surg. 2010;8(5):336-41. . $^{(12)}$

Figura 1. Fluxograma de busca e seleção da amostra final de estudos

Para a análise dos resultados, os artigos selecionados foram agrupados por: autor, ano e país; ob- 
jetivo; nível de evidência; ${ }^{(13)}$ causas do comprometimento do sono e intervençóes de enfermagem na promoçáo do sono. Os resultados foram discutidos à luz dos quatro contextos da experiência humana de conforto (físico, psicoespiritual, social e ambiental) descritos na Teoria de Conforto de Kolcaba. ${ }^{(8)}$

\section{Resultados}

A caracterização dos artigos selecionados para análise revelou que, do total de artigos, quatro $(33,3 \%)$ são provenientes da Austrália, dois (16,7\%) dos Estados Unidos da América e os restantes países possuem apenas uma produção, salientando-se que três $(25 \%)$ são provenientes da Europa. Em relaçáo ao período, verificou-se um predomínio de estudos publicados no ano de 2017 (quatro artigos). O nível de evidência dos artigos é variável com predomínio de estudos de nível 4. ${ }^{(19,22-24)} \mathrm{A}$ análise da evidência disponível nos artigos permitiu identificar diversas causas de interrupção do sono no paciente crítico, nomeadamente: o ruído, a prestação de cuidados, a dor e desconforto, a ventilação mecânica, as emoções negativas, a severidade da condição clínica, a sedaçáo e a luminosidade. Relativamente às intervenções de enfermagem que visam a promoção do sono no paciente em UTI, estas são: a diminuição do ruído e luminosidade, o agrupamento da prestação de cuidados, o controle da dor, a implementação de protocolos, a promoção do ritmo circadiano, o apoio emocional e a formação dos profissionais. Os dados extraídos dos artigos foram apresentados numa tabela-síntese (Quadro 2).

Quadro 2. Síntese dos artigos selecionados na RIL

\begin{tabular}{|c|c|c|c|c|}
\hline $\begin{array}{l}\text { Autores/ } \\
\text { Ano/País }\end{array}$ & Objetivo & $\begin{array}{l}\text { Nível de } \\
\text { evidência }\end{array}$ & $\begin{array}{l}\text { Causas de comprometimento do } \\
\text { sono }\end{array}$ & Intervenções de Enfermagem para promoção do sono \\
\hline $\begin{array}{l}\text { Jones, C., et al. (14) } \\
2012 \\
\text { Reino Unido }\end{array}$ & $\begin{array}{l}\text { Avaliar a utilidade e eficácia das máscaras } \\
\text { de olhos e dos tampões de ouvidos para a } \\
\text { promoção do sono; } \\
\text { Identificar os fatores promotores/ } \\
\text { perturbadores do sono. }\end{array}$ & Nível 3 & $\begin{array}{l}\text { Ruído; Luminosidade; Prestação } \\
\text { de Cuidados; Dor/Desconforto; } \\
\text { Ansiedade. }\end{array}$ & $\begin{array}{l}\text { Utilização de tampões para os ouvidos e máscaras de olhos; } \\
\text { Administração de sedativos e/ou analgesia. }\end{array}$ \\
\hline $\begin{array}{l}\text { Ritmala-Castren M., } \\
\text { et al. }{ }^{(15)} \\
2015 \\
\text { Finlândia } \\
\end{array}$ & $\begin{array}{l}\text { Descrever a qualidade do sono nos } \\
\text { pacientes não-entubados; } \\
\text { Descrever as intervenções de enfermagem } \\
\text { durante o turno da noite na UTI. } \\
\end{array}$ & Nível 2 & Prestação de Cuidados & $\begin{array}{l}\text { Agrupar a prestação de cuidados; Planeamento antecipado dos } \\
\text { cuidados. }\end{array}$ \\
\hline $\begin{array}{l}\text { Beltrami, F., et al. }{ }^{(16)} \\
2015 \\
\text { Brasil }\end{array}$ & $\begin{array}{l}\text { Rever a literatura existente sobre o sono } \\
\text { nas UTIs; } \\
\text { Analisar os métodos de avaliação do sono, } \\
\text { as causas de comprometimento do sono } \\
\text { e as suas implicações na recuperação } \\
\text { do paciente crítico, e as estratégias de } \\
\text { promoção do sono. }\end{array}$ & Nível 5 & $\begin{array}{l}\text { Ruído; Luz; } \\
\text { Prestação de Cuidados; } \\
\text { Fatores intrínsecos ao paciente; } \\
\text { Ventilação Mecânica; } \\
\text { Medicação. }\end{array}$ & $\begin{array}{l}\text { Redução dos níveis de ruído (ajustar os alarmes do monitor e do } \\
\text { ventilador, minimizar a conversa da equipe e fornecer tampões para os } \\
\text { ouvidos); } \\
\text { Redução da luz noturna (diminuir as luzes nos quartos e arredores, } \\
\text { fornecendo máscaras de olhos); } \\
\text { Agrupar a prestação de cuidados; } \\
\text { Melhorar o conforto do paciente (ajustar o ventilador, alívio da dor, } \\
\text { técnicas de relaxamento (massagem ou musicoterapia) e administração } \\
\text { de medicação. }\end{array}$ \\
\hline $\begin{array}{l}\text { Hu, RF., et al. (17) } \\
2015 \\
\text { China }\end{array}$ & $\begin{array}{l}\text { Avaliar a eficácia das medidas não- } \\
\text { farmacológicas na promoção do sono; } \\
\text { Avaliar a eficácia clínica na melhoria da } \\
\text { qualidade do sono e na redução do tempo } \\
\text { de internamento }\end{array}$ & Nível 1 & $\begin{array}{l}\text { Modalidade ventilatória (ex: } \\
\text { assincronias, apneia central ou } \\
\text { esforço respiratório) }\end{array}$ & $\begin{array}{l}\text { Ajustar modalidades e parâmetros ventilatórios, Máscaras de olhos e } \\
\text { tampões ouvidos; } \\
\text { Musicoterapia; } \\
\text { Técnicas de relaxamento (massagens); } \\
\text { Suporte emocional; } \\
\text { Acupressão valeriana. }\end{array}$ \\
\hline $\begin{array}{l}\text { Rittayamai, N., et } \\
\text { al. }{ }^{(18)} \\
2016 \\
\text { Canadá, França e } \\
\text { Tailândia }\end{array}$ & $\begin{array}{l}\text { Rever na literatura os padrões anormais de } \\
\text { sono e alteração do ritmo circadiano, assim } \\
\text { como os efeitos da ventilação mecânica e } \\
\text { sedativos na qualidade e duração do sono } \\
\text { em UTI. }\end{array}$ & Nível 5 & $\begin{array}{l}\text { Ruído; } \\
\text { Ventilação mecânica invasiva; } \\
\text { Apneias de origem central por } \\
\text { excessivo suporte ventilatório } \\
\text { oferecido; } \\
\text { Assincronias paciente-ventilador; } \\
\text { Sedação contínua prolongada. } \\
\end{array}$ & $\begin{array}{l}\text { Minimizar estímulos externos (luzes e ruído dos alarmes); } \\
\text { Vigiar sinais de desadaptação paciente- ventilador; } \\
\text { Ajustar parâmetros ventilatórios; } \\
\text { Conectar o ventilador durante a noite em pacientes traqueostomizados } \\
\text { em fase de desmame. } \\
\text { Promover o ritmo circadiano, interrompendo a sedação contínua durante } \\
\text { o dia. }\end{array}$ \\
\hline $\begin{array}{l}\text { Aitken, L. M., et } \\
\text { al. }{ }^{(19)} \\
2016 \\
\text { Austrália }\end{array}$ & \begin{tabular}{|l|} 
Descrever o autorrelato dos pacientes da \\
UTI sobre o sono durante a permanência na \\
UTI (Questionário RCSQ) e as intervenções \\
por eles sugeridas para promover o sono.
\end{tabular} & Nível 4 & $\begin{array}{l}\text { Dor/ desconforto; Náuseas, vómitos, } \\
\text { diarreia e incontinência; Tosse, sede, } \\
\text { boca seca e fome; Calor/ frio; Ruído } \\
\text { causado pela equipe e equipamentos; } \\
\text { Luminosidade; Prestação de cuidados } \\
\text { de saúde; } \\
\text { Dificuldade em comunicar; } \\
\text { Frustração, medo, preocupações e } \\
\text { pesadelos; } \\
\text { Ansiedade; } \\
\text { Perceção de ambiente não familiar. }\end{array}$ & $\begin{array}{l}\text { Quatro categorias de intervenção: } \\
\text { Farmacológicas (Alívio da dor, utilização de antieméticos, medicação } \\
\text { crónica psiquiátrica e indutora de sono) } \\
\text { Controle Ambiental (Redução do nível de ruído e da luz, reduzir volumes } \\
\text { dos alarmes, oferecer máscaras de olhos e tampões auditivos) } \\
\text { Prestação de Cuidados (Agrupar os cuidados de posicionamento, } \\
\text { higiene e otimização da temperatura); } \\
\text { Dimensão psicossocial (Respeitar rituais e crenças, estabelecer relação } \\
\text { de confiança, musicoterapia). }\end{array}$ \\
\hline
\end{tabular}




\begin{tabular}{|c|c|c|c|c|}
\hline $\begin{array}{l}\text { Autores/ } \\
\text { Ano/País }\end{array}$ & Objetivo & $\begin{array}{l}\text { Nível de } \\
\text { evidência }\end{array}$ & $\begin{array}{l}\text { Causas de comprometimento do } \\
\text { sono }\end{array}$ & Intervenções de Enfermagem para promoção do sono \\
\hline $\begin{array}{l}\text { McAndrew, N., et } \\
\text { al. }{ }^{(20)} \\
2016 \\
\text { Estados Unidos da } \\
\text { América }\end{array}$ & $\begin{array}{l}\text { Perceber o efeito do protocolo Quiet Time } \\
\text { na sedação e sua frequência nos pacientes } \\
\text { críticos e ainda no nível de delirium. } \\
\text { Determinar se consecutivos Quiet Time } \\
\text { influenciam a nível fisiológico }\end{array}$ & Nível 2 & $\begin{array}{l}\text { Ventilação mecânica invasiva } \\
\text { (21,3\%); Prestação de cuidados de } \\
\text { saúde (55,2\%); outros procedimentos } \\
(7,6 \%) ; \text { ruído }(5,2 \%) \text {, mudança no } \\
\text { estado clínico do paciente }(4,5 \%) \text {. }\end{array}$ & $\begin{array}{l}\text { Protocolo Quiet Time: } \\
\text { Apagar as luzes do quarto, fechar a porta do quarto e cortinas; desliga } \\
\text { a televisão, agrupar a prestação de cuidados. }\end{array}$ \\
\hline $\begin{array}{l}\text { Boyko, Y.; et al. (21) } \\
2017 \\
\text { Dinamarca }\end{array}$ & $\begin{array}{l}\text { Determinar o papel do ambiente das UTIS } \\
\text { na melhoria da qualidade do sono nos } \\
\text { pacientes ventilados; } \\
\text { Comparar os padrões de sono em pacientes } \\
\text { ventilados e orientados durante 48h com o } \\
\text { protocolo Quiet Routine VS rotina usual. }\end{array}$ & Nível 2 & $\begin{array}{l}\text { Ruído (os níveis de ruído na UTI são } \\
\text { superiores aos recomendados pela } \\
\text { OMS). }\end{array}$ & $\begin{array}{l}\text { Protocolo Quiet Routine: } \\
\text { Inexistência de visitas durante a noite das 22h - 06h, diminuição } \\
\text { dos alarmes e da luminosidade, ausência de conversas nos quartos, } \\
\text { intervenções de saúde estritamente necessárias e equipe de } \\
\text { enfermagem num posto de observação. }\end{array}$ \\
\hline $\begin{array}{l}\text { Ding, Q., et al. } 22 \text { ) } \\
2017 \\
\text { Estados Unidos da } \\
\text { América }\end{array}$ & $\begin{array}{l}\text { Explorar as perceções e crenças dos } \\
\text { profissionais, pacientes e acompanhantes } \\
\text { acerca dos fatores ambientais e não } \\
\text { ambientais que afetam o sono dos } \\
\text { pacientes numa UTI. }\end{array}$ & Nível 4 & $\begin{array}{l}\text { Ruído; interrupções frequentes no } \\
\text { quarto; fluxo de trabalho imprevisível; } \\
\text { dor/desconforto; ansiedade, } \\
\text { problemas crónicos em dormir, } \\
\text { doença. }\end{array}$ & $\begin{array}{l}\text { Reduzir o ruído, fechar a cortina e a porta para reduzir a } \\
\text { luminosidade, agrupar a prestação de cuidados de saúde; gestão } \\
\text { emocional dos pacientes e estabelecimento de uma relação de } \\
\text { proximidade com os profissionais de saúde; promover o ritmo } \\
\text { circadiano mantendo os pacientes acordados durante o dia, educação } \\
\text { sobre os tópicos relacionados com o sono na UTI; promover o conforto } \\
\text { do paciente. }\end{array}$ \\
\hline $\begin{array}{l}\text { Menear, A., et al. }{ }^{(23)} \\
2017 \\
\text { Austrália }\end{array}$ & $\begin{array}{l}\text { Estudar a qualidade do sono dos pacientes } \\
\text { internados em UTI e comparar o uso das } \\
\text { estratégias promotoras do sono utilizadas } \\
\text { na unidade com estudos anteriores; } \\
\text { Identificar os fatores inibidores do sono e as } \\
\text { estratégias de promoção do mesmo. } \\
\end{array}$ & Nível 4 & $\begin{array}{l}\text { Dor/desconforto, cuidados de saúde } \\
\text { ao paciente prestados durante a } \\
\text { noite. }\end{array}$ & $\begin{array}{l}\text { Adotar protocolos de promoção do sono; valorizar os rituais de sono } \\
\text { do paciente; fornecer máscaras de olhos e tampões de ouvidos; } \\
\text { agrupar a prestação de cuidados de saúde ao paciente; gerir o regime } \\
\text { medicamentoso. }\end{array}$ \\
\hline $\begin{array}{l}\text { Delaney, L., et al. (24) } \\
2017 \\
\text { Austrália }\end{array}$ & $\begin{array}{l}\text { Investigar a intensidade do ruído noturno } \\
\text { na UTI como fator perturbador do sono, } \\
\text { comparando com o estipulado pela WHO e } \\
\text { comparar a intensidade do ruído em open } \\
\text { space e quarto de isolamento. }\end{array}$ & Nível 4 & $\begin{array}{l}0 \text { ruído no período noturno excedeu } \\
\text { as recomendações internacionais } \\
\text { ao longo de toda a monitorização, } \\
\text { mesmo os valores de ruído mínimos; } \\
\text { Imprevisibilidade do ruído ambiental. }\end{array}$ & $\begin{array}{l}\text { Regular alarmes de monitores; ensinar os profissionais acerca das } \\
\text { consequências psicológicas do ruído na recuperação do paciente: } \\
\text { oferecer tampões para ouvidos; redesenhar o ambiente da UTI } \\
\text { (materiais que absorvam o som e estruturas que permitam a exposição } \\
\text { solar). }\end{array}$ \\
\hline $\begin{array}{l}\text { Knauert M. P., et } \\
\text { al. (25) } \\
2018 \\
\text { Austrália }\end{array}$ & $\begin{array}{l}\text { Identificar as fontes de comprometimento } \\
\text { do sono do paciente durante a noite; } \\
\text { Avaliar e rever o protocolo Naptime e } \\
\text { adaptá-lo a todas as unidades. }\end{array}$ & Nível 3 & $\begin{array}{l}\text { Ruído; dor; ansiedade ou tristeza; } \\
\text { Prestação de cuidados. }\end{array}$ & $\begin{array}{l}\text { Agrupar a prestação de cuidados ao paciente durante o protocolo } \\
\text { Naptime; utilizar lanternas, luzes noturnas e falar em voz baixa; fechar } \\
\text { as cortinas e portas antes do Naptime; manter as cortinas da janela } \\
\text { abertas durante o dia para promover o ritmo circadiano; ajustar os } \\
\text { alarmes (ventiladores, bombas infusoras e monitores). }\end{array}$ \\
\hline
\end{tabular}

\section{Discussão}

\subsection{Causas de Comprometimento do Sono em Unidade de Terapia Intensiva}

O ruído foi identificado como sendo uma das principais causas de comprometimento do sono na UTI, ${ }^{(14,16,18-22,24,25)}$ podendo ter várias fontes, como os alarmes de bombas infusoras, monitores e ventiladores, as conversas entre membros da equipe multiprofissional, os telefones, a televisão, as portas em funcionamento e ainda a prestaçáo de cuidados a outros pacientes. De acordo com Delaney et al., ${ }^{(24)}$ o ruído excedeu as recomendaçóes da World Health Organization (WHO) durante toda a monitorização, sendo identificada uma grande variabilidade de picos de ruído ao longo da noite até mesmo nos quartos individuais porque, muitas vezes, os profissionais aumentam o volume dos alarmes para os conseguirem ouvir do lado de fora. No entanto, outros artigos analisados, apesar de concluírem o ruído como causa de comprometimento do sono, não o consideram um fator maior, pois este só foi mencionado pelos pacientes numa percentagem de $5,2 \%$ face aos outros fatores, ${ }^{(20)}$ e apenas $10-30 \%$ dos despertares podem ser atribuídos ao ruído ambiental, ${ }^{(16)}$ o que nestes dois estudos contraria a evidência geral.

A prestaçáo de cuidados de saúde ao paciente é a segunda principal causa de comprometimento do sono, ${ }^{(14-16,19-20,23,25)}$ apesar de dois estudos referirem ser a causa mais relatada pelos pacientes. ${ }^{(20,23)}$ Em contraste com estes resultados, Beltrami et al. ${ }^{(16)}$ chegaram a duas conclusóes opostas: um dos estudos revistos refere que no turno da noite, observaram 9 períodos de prestação de cuidados, de 2 a 3 horas cada, logo durante as 147 noites em que decorreu o estudo, observou-se que apenas $6 \%$ do período do turno da noite esteve disponível para o sono. Em contraste, concluem também que apesar de os cuidados de saúde serem uma causa de interrupçáo do sono, através da prestação de cuidados de higiene oral e ocular, posicionamentos, adminis- 
tração de terapêutica e coleta de sangue, estas intervençôes de enfermagem não parecem ser a principal fonte de distúrbios do sono em pacientes internados em UTIs, correspondendo apenas a $20 \%$ dos despertares noturnos. ${ }^{(16)}$ As intervençóes de enfermagem numa UTI, durante a prestação de cuidados, devido à especificidade do contexto requerem uma manipulação de elementos naturais e artificiais do ambiente, tais como os meios tecnológicos, causando desconforto ambiental no paciente.

Um outro motivo de comprometimento do sono abordado nos estudos foi a dor e o desconforto, ${ }^{(14,19,22,23,25)}$ sendo que num dos estudos esta é a causa mais relatada pelos pacientes. ${ }^{(23)}$ Para além disso, também num dos estudos, mais de 50\% dos profissionais de saúde relataram que o desconforto e dor contribuem para as anormalidades no sono. ${ }^{(22)}$

O comprometimento do sono, associado à ventilação mecânica, surge devido às apneias de causa central geradas por ventilação excessiva, por pressão de suporte demasiado alta, e também devido à existência de assincronias ventilatórias. ${ }^{(16-18,20)}$ Além disso, está presente também o desconforto do tubo endotraqueal, a aspiração de secreçôes e o reposicionamento frequente, como potenciadores da má qualidade do sono. ${ }^{(16)}$

A vivência de emoçóes, como ansiedade, tristeza, medo, frustração e preocupação, durante a internação em UTI comprometem o seu padrão de sono. ${ }^{(14,19,22,25)}$ Segundo Ding et al., ${ }^{(22)}$ os pacientes atribuem maior importância à ansiedade e às emoçóes como fatores que comprometem o sono devido, frequentemente, à incerteza e preocupação sobre o seu estado de saúde, assim 57\% dos pacientes entrevistados afirmaram que os fatores emocionais e cognitivos afetam mais a qualidade do sono do que o ambiente da UTI. Noutro estudo, quer os pacientes, quer os profissionais de saúde identificaram as emoçôes e a insegurança como fatores preponderantes na interrupção do sono. ${ }^{(25)}$

A severidade da condição clínica do paciente pode estar associada à diminuição da quantidade e da qualidade do sono. ${ }^{(16,20,22)}$ No pós-operatório imediato de uma grande cirurgia, o sono profundo está reduzido ou ausente, ${ }^{(16)}$ além de que os pacientes internados em UTIs podem ter uma doença preexistente que contribui para a má qualidade do sono. ${ }^{(22)}$ Um estudo recente concluiu que ser mulher, ter idade superior a 55 anos, consumir bebidas alcoólicas, usar substâncias ilícitas, ter antecedente de angina de peito, obesidade e depressão são fatores predisponentes para alteraçóes na qualidade e duração de sono. ${ }^{(26)}$ Por outro lado, a necessidade de sedação contínua pode alterar o ritmo circadiano do sono, sendo que estas alteraçóes dependem também do tipo de sedativo utilizado. ${ }^{(16,18)}$

Em relação à regulação da luminosidade, esta contribui para a adequação do ritmo circadiano do paciente, contudo as luzes são acesas ininterruptamente provocando distúrbios do sono, ${ }^{(14,19)}$ até quando são prestados cuidados durante a noite, onde a luminosidade é essencial para a obtenção de colheitas para exames laboratoriais. ${ }^{(16)}$

O desconforto ambiental é a principal causa de distúrbio do sono (ruído, prestação de cuidados e luminosidade), seguido do desconforto físico (dor, ventilação mecânica, severidade da condição clínica e sedação) e finalmente surge o desconforto psicoespiritual e social (emoçốes negativas). A experiência de conforto é um fenômeno intersubjetivo, sendo uma meta para a qual contribuem os comportamentos dirigidos à saúde adotados pelo paciente e/ou enfermeiro. ${ }^{(8)}$ Nesta perspectiva, a análise dos resultados orienta a prática de enfermagem para considerar de forma reflexiva a sua ação, face aos contextos de desconforto, sobre como podem ser favorecidos para retroalimentar o estado de conforto melhorado.

\subsection{Intervenções de Enfermagem para a Promoção do Sono em UTI}

Relativamente às intervenções de enfermagem, destaca-se a redução geral do ruído, ${ }^{(14,16,18-25)}$ sendo que as estratégias referidas contemplam o ajuste dos alarmes dos monitores e dos ventiladores, o minimizar a conversa entre a equipe, mas também vários estudos sugerem o uso de tampóes para os ouvidos. ${ }^{(14,16,17,19,23,24)} \mathrm{A}$ este nível é interessante referir que nem todos os estudos que sugerem o uso de tampóes de ouvidos defendem a redução do ruído ambiental, ${ }^{(17,23)}$ assumindo a sua presença como algo imutável nestes contextos.

No âmbito do conforto ambiental emerge ainda a redução da luminosidade no período noturno, ${ }^{(16,18,20-22,25)}$ sendo que os níveis de luz podem ser 
reduzidos através da diminuição das luzes no serviço, mas também fornecendo máscaras de olhos. ${ }^{(14,16-17,19,23)}$ Assim, os pacientes a quem forneceram máscaras de olhos e tampóes auditivos relataram melhor qualidade do sono do que aqueles que receberam o tratamento padrão e para além disso, também os pacientes que utilizaram apenas tampóes de ouvidos relataram ter uma melhor qualidade de sono, em comparação com o grupo controle. ${ }^{(14,17)} \mathrm{O}$ uso de máscaras e tampóes serve como lembrança para os profissionais de saúde da importância do sono e aumenta a sensação de controle dos pacientes, reduzindo desta forma a ansiedade relativa a um ambiente estranho e stressante.

A promoção do sono na UTI pode ser alcançada através do agrupamento da prestação de cuidados de saúde ao paciente de acordo com alguns estudos. $^{(15,16,19,20,22,25)}$ Determinadas atividades de cuidados devem ser planejadas no tempo de forma a evitar interrupçóes desnecessárias do sono. Os pacientes que foram submetidos a um maior número de cuidados de enfermagem não agrupados no tempo tiveram um maior período de sono superficial. ${ }^{(15)}$ Por outro lado, a concentração da prestação dos cuidados, no período diurno, contribui para a promoçáo do ritmo circadiano e simultaneamente diminui da ansiedade do paciente associada à monitorização dos profissionais de saúde. ${ }^{(22)}$

Relativamente ao conforto físico do paciente, alcançado pela gestão das sensaçôes corporais, através do controle da dor, em UTI destacam-se medidas farmacológicas (analgésicos, sedativos, psicotrópicos, antieméticos e medicação crónica do paciente) e não farmacológicas (posicionamentos, cuidados de higiene, musicoterapia, massagens e otimização da sincronia paciente-ventilador). ${ }^{(14,16-19,22,23)}$

Salienta-se a criação e implementação de protocolos específicos que visam a promoção do sono nestes contextos com enfoque no período noturno, baseando-se sobretudo em intervençôes de enfermagem na redução do ruído e luminosidade. ${ }^{(15,20,21,23,25)}$ A aplicação dos protocolos contribui para a diminuição da necessidade de medicamentos sedativos, o que consequentemente pode aumentar a probabilidade de sucesso do desmame ventilatório e ainda diminuir o tempo de internação. ${ }^{(20)}$ É importante uma adaptação individual do protocolo, baseado nas necessidades de cada paciente e nos recursos de cada UTI. ${ }^{(25)}$

A promoção do ritmo circadiano é uma intervenção que surge da produção científica, quer através da manutenção dos pacientes num estado vígil no período diurno, ${ }^{(18,22)}$ quer através da exposição solar dos pacientes no período diurno. ${ }^{(24,25)}$

Por fim, relativamente à dimensão psicoespiritual e social do conforto, na promoção do sono emerge o apoio emocional através do estabelecimento de uma relação de confiança, do respeito pelos rituais e crenças do paciente. ${ }^{(19)}$ A referir a importância da gestão emocional dos pacientes, pois 50\% dos pacientes admitem que uma relação próxima com os profissionais de saúde pode melhorar a qualidade do sono. ${ }^{(22)}$ Ainda relativamente à dimensão do conforto social, face à necessidade de manutenção de relações interpessoais significativas, a destacar o papel ambivalente que as visitas familiares assumem no sono dos pacientes, porque por um lado, as visitas são encaradas como um fator perturbador do sono e como tal, são adotadas medidas de restrição de visitas durante a noite. ${ }^{(21,25)}$ Por outro lado, as visitas familiares são encaradas como medidas promotoras do sono, na medida em que oferecem suporte emocional aos pacientes e diminuem a ansiedade dos mesmos o que, consequentemente, conduz a uma melhoria da qualidade e da quantidade de sono, observando-se um aumento significativo do número total de horas dormidas, no grupo onde se alargou o horário das visitas até ao momento do jantar. ${ }^{(17)}$

A importância da educação dos profissionais de saúde acerca das consequências da privação do sono e da importância do seu papel na promoção do mesmo, bem como intervençóes possíveis de adotar é um aspecto que apenas é enfatizado em dois dos estudos consultados, ${ }^{(22,24)}$ sendo pertinente articular este achado com a dificuldade de implementaçáo de protocolos específicos para a promoção do sono.

\section{Conclusão}

O comprometimento do sono, enquanto necessidade humana básica, do paciente em UTI é influenciado sobretudo por fatores ambientais, tendo reper- 
cussóes na melhoria do quadro clínico e no contexto da experiência de conforto ambiental, segundo a teoria de conforto de Kolcaba. A partir da revisão integrativa da literatura realizada conclui-se que existe uma maior valorização dos fatores ambientais como fatores inibidores do sono em detrimento da dimensão emocional do paciente crítico. Torna-se, deste modo, importante existir mais investigação relativa ao impacto da dimensão emocional no sono nas UTIs, promovendo o conforto psicoespiritual. Relativamente às intervençóes, na maioria dos artigos analisados, estas vão ao encontro da gestão do conforto ambiental e físico, porém, a sua eficácia na prática para a melhoria da quantidade e qualidade de sono dos pacientes, nem sempre é consensual. Observou-se também que o contexto da experiência do conforto social do paciente para o sono é pouco abordado nos artigos, alertando para a necessidade de existir mais investimento futuro nesta dimensão, promovendo desta forma uma visão holística da pessoa, considerando a família como parceira e foco de cuidados. Assim, considerando as lacunas identificadas na discussão dos resultados e suas implicaçóes para a prática de enfermagem, sugerem-se novos estudos, minimizando as lacunas existentes na literatura.

\section{Referências}

1. Weinhouse GL, Schwab RJ, Watson PL, Patil N, Vaccaro B, Pandharipande $P$, et al. Bench-to-bedside review: delirium in ICU patients - importance of sleep deprivation. Crit Care. 2009;13(6):234.

2. Conselho Internacional de Enfermeiros (ICN). Ordem dos Enfermeiros $(\mathrm{OE})$, editores. Classificação Internacional para a prática de Enfermagem: CIPE Versão 2015. Loures: Lusodidacta; 2016. 278p.

3. Urden LD, Stacy KM, Lough ME, editors. Critical care nursing: diagnosis and management. 8th ed. Missouri: Elsevier; 2018. 1049p.

4. Pisani MA, Friese RS, Gehlbach BK, Schwab RJ, Weinhouse GL, Jones SF. Sleep in the intensive care unit. Am J Respir Crit Care Med. 2015 Apr;191(7):731-8.

5. Faria R, Moreno RP. Delirium na unidade de cuidados intensivos: uma realidade subdiagnosticada. Rev Bras Ter Intensiva. 2013;25(2):13747.

6. Portugal. Lei n. $^{0} 156 / 2015$, de 16 de Setembro de 2015. Estatuto da Ordem dos Enfermeiros. Diário da República n. ${ }^{0}$ 181/2015; Série l:8059-8105.

7. Tomey AM, Alligood MR. Teóricas de enfermagem e a sua obra: modelos e teorias de enfermagem. 5th ed. Lisboa: Lusodidacta; 2004. $766 \mathrm{p}$.
8. Kolcaba K. Confort Theory and Practice: A vision for holistic health care and research. 1st ed. New York: Springer Publishing Company; 2003. $276 \mathrm{p}$.

9. Elliott R, McKinley S, Cistulli P, Fien M. Characterisation of sleep in intensive care using 24-hour polysomnography: an observational study. Crit Care. 2013;17(2):R46.

10. Mendes K, Silveira R, Galvão C. Revisão integrativa: método de pesquisa para a incorporação de evidências na saúde e na enfermagem. Texto Contexto Enferm. 2008;17(4):758-64.

11. Cronin $P$, Ryan $F$, Coughlan M. Undertaking a literature review: a stepby-step approach. Br J Nurs. 2008;17(1):38-43.

12. Moher D, Liberati A, Tetzlaff J, Altman DG; PRISMA Group. Preferred reporting items for systematic reviews and meta-analyses: the PRISMA statement. Int J Surg. 2010;8(5):336-41.

13. Oxford Centre for Evidence-based Medicine (CEBM). Levels of evidence [Internet]. Oxford: CEBM; 2009. [cited 2019 0ct 28]. Available from: http://www.cebm.net/oxford-centre-evidence-based-medicine-levelsevidence-march-2009

14. Jones C, Dawson D. Eye masks and earplugs improve patient's perception of sleep. Nurs Crit Care. 2012;17(5):247-54.

15. Ritmala-Castren M, Virtanen I, Leivo S, Kaukonen KM, Leino-Kilpi H. Sleep and nursing care activities in an intensive care unit. Nurs Health Sci. 2015;17(3):354-61.

16. Beltrami FG, Nguyen XL, Pichereau C, Maury E, Fleury B, Fagondes S. Sleep in the intensive care unit. J Bras Pneumol. 2015;41(6):539-46.

17. Hu RF, Jiang XY, Chen J, Zeng Z, Chen XY, LiY, et al. Non-pharmacological interventions for sleep promotion in the intensive care unit. Cochrane Database Syst Rev. 2015 0ct;(10):CD008808.

18. Rittayamai N, Wilcox E, Drouot X, Mehta S, Goffi A, Brochard L. Positive and negative effects of mechanical ventilation on sleep in the ICU: a review with clinical recommendations. Intensive Care Med. 2016;42(4):531-41.

19. Aitken LM, Elliott R, Mitchell M, Davis C, Macfarlane B, Ullman A, et al. Sleep assessment by patients and nurses in the intensive care: an exploratory descriptive study. Aust Crit Care. 2017;30(2):59-66.

20. McAndrew NS, Leske J, Guttormson J, Kelber ST, Moore K, Dabrowski S. Quiet time for mechanically ventilated patients in the medical intensive care unit. Intensive Crit Care Nurs. 2016;35:22-7.

21. Boyko Y, Jennum P, Nikolic M, Holst R, Oerding H, Toft P. Sleep in intensive care unit: the role of environment. J Crit Care. 2017;37:99105.

22. Ding Q, Redeker NS, Pisani MA, Yaggi HK, Knauert MP. Factors influencing patients sleep in the intensive care unit: perceptions of patients and clinic staff. Am J Crit Care. 2017;26(4):278-86.

23. Menear A, Elliott R, M Aitken L, Lal S, McKinley S. Repeated sleepquality assessment and use of sleep-promoting interventions in ICU. Nurs Crit Care. 2017 Nov;22(6):348-54.

24. Delaney LJ, Currie MJ, Huang HC, Lopez V, Litton E, Van Haren F. The nocturnal acoustical intensity of the intensive care environment: an observational study. J Intensive Care. 2017;5(41):41.

25. Knauert MP, Redeker NS, Yaggi HK, Bennick M, Pisani MA. Creating a naptime: an overnight, nonpharmacologic intensive care unit sleep promotion protocol. J Patient Exp. 2018;5(3):180-7.

26. Simões ND, Monteiro LH, Lucchese R, Amorim TA, Denardi TC, Vera I, et al. Qualidade e duração de sono entre usuários da rede pública de saúde. Acta Paul Enferm. 2019;32(5):530-7. 